Journal of Engineering and Applied Sciences 5 (6): 382-387, 2010

ISSN: 1816-949X

(C) Medwell Journals, 2010

\title{
Enhanced Path Roperties of Brownian Motion
}

\author{
R. Sasikumar \\ Department of Statistics, Manonmaniam Sundaranar University, 627012 Tirunelveli, India
}

\begin{abstract}
Human lives are full of uncertainties as with many natural phenomena. No one can precisely foresee what will happen in the next second, minute and so on. The sample realization of a stochastic process are vital from the point of view of Brownian path analysis. A Brownian Motion (BM) with drift zero and diffusion coefficient, one is defined as standard BM whose path properties are used for analysis in several physical and chemical problems. Rather than accepting the fact that the future is always uncertain, many models and algorithms have been continuously formulated for the prediction of matters involving uncertain elements. One of them is the Brownian model. In this study, we discuss the enhanced path properties of a one dimensional standard Brownian motion $\mathrm{B}(\mathrm{t}), \mathrm{t} \geq 0$ on a complete probability space $(\Omega, \mathrm{F}, \mathrm{P})$. The law of the iterated logarithm about how Brownian Motion (BM) oscillates in a neighbourhood of the origin is also discussed.
\end{abstract}

Key words: Brownian motion, brownian paths, bounded variation, local maximum, borel-cantelli lemma, India

\section{INTRODUCTION}

The physical origins of the Brownian motion process suggest that the possible realizations $\mathrm{B}(\mathrm{t})$ as the graphs of the $\mathrm{x}$ coordinates of the position of a particle (i.e., the sample paths) whose movements result from continuous collisions in the surrounding medium are continuous functions. A complete description of the path structure of the BM process can be found by Levy (1965) and Ito and McKean (1965).

The paths of $\mathrm{BM}$ processes may intriguing and bizarre properties, they are continuous but badly behaved. We start by showing that the paths are nowhere differentiable. It is convenient to assume that the probability space is complete which means that all subsets of events of probability 0 are events. This is no loss of generality.

Theorem: Almost every paths of $\mathrm{BM}$ are nowhere differentiable.

Proof: We shall prove stronger results that $B(t)$ has no point increase or decrease. Fix $\beta>0$ and suppose that $B(t)$ has derivative $\mathrm{B}^{\prime}(\mathrm{s}),\left|\mathrm{B}^{\prime}(\mathrm{s})\right|<\beta$ at some point $\mathrm{s} \in[0,1]$ then there is an $n_{0}$ such that for $n>n_{0}$ :

$$
|\mathrm{B}(\mathrm{t})-\mathrm{B}(\mathrm{s})| \leq 2 \mathrm{~b}|\mathrm{t}-\mathrm{s}| \text { if }|\mathrm{t}-\mathrm{s}| \leq 2 / \mathrm{n}
$$

Let $B\left(\right.$.) denote functions on $[0,1] . A_{n}=\{B($.$) ; there$ exists s such that:

$$
|\mathrm{B}(\mathrm{t})-\mathrm{B}(\mathrm{s})| \leq 2 \mathrm{~b}|\mathrm{t}-\mathrm{s}| \text { if }|\mathrm{t}-\mathrm{s}| \leq 2 / \mathrm{n}\}
$$

Then $A_{n} \uparrow A \subset$ s set of all sample paths of $B(t)$ on $[0,1]$ having a derivative at any point which is $<\beta$ in absolute value\}. If Eq. 1 holds and $\mathrm{k}$ be the largest integer such that $\mathrm{k} / \mathrm{n} \leq \mathrm{s}$, then the following is implied:

$$
Y_{n}=\max \left(\begin{array}{l}
\left|B\left(\frac{k+2}{n}\right)-B\left(\frac{k+1}{n}\right)\right|, \\
\left|B\left(\frac{k+1}{n}\right)-B\left(\frac{k}{n}\right)\right|, \\
\left|B\left(\frac{k}{n}\right)-B\left(\frac{k-1}{n}\right)\right|
\end{array}\right) \leq 6 b / n
$$

Therefore, if $W_{n}=\left\{B(\right.$.$\left.) ; at least one Y_{k} \leq 6 \beta / n\right\}$, then:

$$
\mathrm{A}_{\mathrm{n}} \subset \mathrm{W}_{\mathrm{n}}=\bigcup_{\mathrm{k}=1}^{\mathrm{n}-2}\left\{\mathrm{~B}(.) ; \mathrm{Y}_{\mathrm{k}} \leq 6 \mathrm{~b} / \mathrm{n}\right\}
$$

$$
\mathrm{P}\left(\mathrm{W}_{\mathrm{n}}\right) \leq \sum_{\mathrm{k}=1}^{\mathrm{n}-2} \mathrm{P}(\max )\left|\mathrm{B}\left(\frac{\mathrm{k}+2}{\mathrm{n}}\right)-\mathrm{B}\left(\frac{\mathrm{k}+1}{\mathrm{n}}\right)\right|,
$$$$
\left|\begin{array}{c}
B\left(\frac{k+1}{n}\right) \\
-B\left(\frac{k}{n}\right)
\end{array}\right|\left|B\left(\frac{k}{n}\right)-B\left(\frac{k-1}{n}\right)\right| \leq\left(\frac{6 b}{n}\right)
$$

$\leq \mathrm{nP}(\max )\left|\mathrm{B}\left(\frac{3}{\mathrm{n}}\right)-\mathrm{B}\left(\frac{2}{\mathrm{n}}\right)\right|,\left|\mathrm{B}\left(\frac{2}{\mathrm{n}}\right)-\mathrm{B}\left(\frac{1}{\mathrm{n}}\right)\right|,\left|\mathrm{B}\left(\frac{1}{\mathrm{n}}\right)\right| \leq\left(\frac{6 \mathrm{~b}}{\mathrm{n}}\right)$ 


$$
\begin{aligned}
& =\mathrm{nP}^{3}\left(\left|\mathrm{X}\left(\frac{1}{\mathrm{n}}\right) \leq \frac{6 \beta}{\mathrm{n}}\right|\right)=\mathrm{n}\left[\sqrt{\frac{\mathrm{n}}{2 \pi}} \int_{-6 \beta / \mathrm{n}}^{6 \beta / \mathrm{n}} \mathrm{e}^{-\mathrm{nx}^{2} / 2} \mathrm{dx}\right]^{3} \\
& =\mathrm{n}\left[\frac{1}{\sqrt{2 \pi \mathrm{n}}} \int_{-6 \beta}^{6 \beta} \mathrm{e}^{-\mathrm{nx} 2 / 2} \mathrm{dy}\right]^{3},(\mathrm{y}=\mathrm{nx}) \rightarrow 0
\end{aligned}
$$

Therefore, $P(A)=\lim _{n \rightarrow \infty} P\left(A_{n}\right) \leq \lim _{n \rightarrow \infty} P\left(W_{n}\right)=0$ which implies the theorem.

\section{THE VARIATION OF BROWNIAN PATHS}

A real valued function $\mathrm{f}$ with domain $[0,1]$ has bounded variation if it is rectifiable that is the graph has finite length. This means that:

$$
\sup \sum_{i=1}^{\mathrm{n}}\left|\mathrm{f}\left(\mathrm{t}_{\mathrm{i}}\right)-\mathrm{f}\left(\mathrm{t}_{\mathrm{i}-1}\right)\right|<\infty
$$

Where, the sup is taken over all finite partitions of $[0,1]$ of the form $0 \leq t_{0} \leq t_{1} \leq \ldots \leq t_{n}=1$ and where we allow $n$ and $t_{1}, \ldots, t_{n}$ to vary. A well known result from analysis is that a function which has bounded variation is almost everywhere differentiable.

Since, we know from the previous result that almost no Brownian Path (BP) is differentiable anywhere, we conclude that Brownian paths do not have bounded variation.

Result: Almost no path of BM has bounded variation. Therefore, almost no path is rectifiable.

\section{QUADRATIC VARIATION}

Brownian paths are badly behaved continuous functions. They are neither differentiable nor rectifiable. In trying to calculate the length of a path $B(t), 0 \leq t \leq 1$, we choose division points for $[0,1]$ which we call:

$$
\Pi=\left\{0=\mathrm{t}_{0}<\mathrm{t}_{1}<\ldots<\mathrm{t}_{\mathrm{n}}=1\right\}
$$

An important characteristic of the division points 2 is how far apart any two successive points can be so, we define:

$$
\Delta(\Pi)=\max _{1 \leq \mathrm{k} \leq \mathrm{n}}\left|\mathrm{t}_{\mathrm{k}}-\mathrm{t}_{\mathrm{k}-1}\right|
$$

Any two successive division points are most $\Delta(\Pi)$ units apart. To calculate the length of the path, we compute:

$$
\sum_{\mathrm{k}=1}^{\mathrm{n}} \mathrm{B}\left(\mathrm{t}_{\mathrm{k}}, \mathrm{w}\right)-\mathrm{B}\left(\mathrm{t}_{\mathrm{k}-1}, \mathrm{w}\right)
$$

and let $\Delta(\boldsymbol{I})$ go to zero. The resulting limit is almost surely infinite. If $\Delta(I I)$ is small, each individual difference $\left|\mathrm{B}\left(\mathrm{t}_{\mathrm{k}}, \mathrm{w}\right)-\mathrm{B}\left(\mathrm{t}_{\mathrm{k}-1}, \mathrm{w}\right)\right|$ is small by continuity but not small enough to make the sums converge. If we make the individual difference samller by say squaring each then there is a chance the limit will be finite. Thus, we define:

$$
\mathrm{Q}(\Pi, \mathrm{w})=\sum_{\mathrm{k}=1}^{\mathrm{n}}\left|\mathrm{B}\left(\mathrm{t}_{\mathrm{k}}, \mathrm{w}\right)-\mathrm{B}\left(\mathrm{t}_{\mathrm{k}-1}, \mathrm{w}\right)\right|^{2}
$$

and call $\mathrm{Q}(\mathrm{I}, \mathrm{w})$ the quadratic variation of $\{\mathrm{B}(\mathrm{t}, \mathrm{w}), 0 \leq \mathrm{t}<1\}$ over $I$. If the limit exists as $\Delta \rightarrow 0$, we call the limit the quadratic variation of $\mathrm{B}$ on $[0,1]$. If instead of $[0,1]$ we had taken $[0, t]$ for $t>0$, then the quadratic variation of $B$ on $[0, t]$ would be a function of $t$ and hence, a stochastic process which is called the quadratic variation process. In the theory of stochastic integration, such processes are important as increasing processes which can be subtracted from sub martingales to give martingales. Quadratic variation processes are also used as random time change which turn processes constructed from Brownian motions using stochastic integration into new Brownian motions.

Theorem: If $\Pi^{(i)} \subset \Pi^{(1+1)}$ and $\Delta\left(\Pi^{(i)}\right) \rightarrow 0$ fast enough, then almost surely $Q\left(\Pi^{(i)}\right) \rightarrow 1$ which is the length of the interval we have decomposed (If instead of decomposing $[0,1]$, we decompose $[0, t]$, then the limit would be $t)$. Fancier versions of this result exist; the present statement and proof, modeled after the treatment in BM, 1968 are given because of their simplicity for example, McKean (1969), Doob (1953) and Karatzas and Shreve (1988). Before giving the proof, we need to recall the following simple fact.

Lemma: Let $\mathrm{N}$ be a $\mathrm{N}(0,1)$ random variable. Then, the moment generating function is:

$$
\mathrm{Ee}^{\alpha \mathrm{N}}=\mathrm{e}^{\alpha^{2} / 2},-\infty<\alpha<\infty,
$$

and;

$$
\mathrm{E}\left(\mathrm{N}^{4}\right)=3
$$

Proof of the theorem: We begin by observation that if:

then:

$$
\Pi^{(1)}=\left\{0=\mathrm{t}_{0}<\mathrm{t}_{1}<\ldots<\mathrm{t}_{\mathrm{n}}=\mathrm{n}\right\}
$$




$$
\begin{aligned}
\mathrm{Q}\left(\Pi^{(i)}\right)-1= & \sum_{\mathrm{i}=1}^{\mathrm{n}}\left\{\left|\mathrm{B}\left(\mathrm{t}_{\mathrm{i}}\right)-\mathrm{B}\left(\mathrm{t}_{\mathrm{i}-1}\right)\right|^{2}-\left(\mathrm{t}_{\mathrm{i}}-\mathrm{t}_{\mathrm{i}-1}\right)\right\} \\
& \sum_{\mathrm{i}=1}^{\mathrm{n}} \theta_{\mathrm{i}}
\end{aligned}
$$

Where, $\theta_{\mathrm{i}}=\left\{\left|\mathrm{B}\left(\mathrm{t}_{\mathrm{i}}\right)-\mathrm{B}\left(\mathrm{t}_{\mathrm{i}-1}\right)\right|^{2}-\left(\mathrm{t}_{\mathrm{i}}-\mathrm{t}_{\mathrm{i}-1}\right)\right\}$ forms a sequence of independent, zero mean random variables. Because the variance of a sum of independent random variables is the sum of the variances, we have:

$$
\begin{gathered}
\mathrm{E}\left(\mathrm{Q}\left(\Pi^{(\mathrm{i})}\right)-1\right)^{2}=\mathrm{E}\left(\sum_{\mathrm{i}=1}^{\mathrm{n}} \theta_{\mathrm{i}}\right)^{2}=\sum_{\mathrm{i}=1}^{\mathrm{n}} \mathrm{E} \theta_{\mathrm{i}}^{2} \\
\sum_{\mathrm{i}=1}^{\mathrm{n}} \mathrm{E}\left(\left(\mathrm{B}\left(\mathrm{t}_{\mathrm{i}}\right)-\mathrm{B}\left(\mathrm{t}_{\mathrm{i}-1}\right)\right)^{4}-2\left(\mathrm{~B}\left(\mathrm{t}_{\mathrm{i}}\right)-\right.\right. \\
\left.\mathrm{B}\left(\mathrm{t}_{\mathrm{i}-1}\right)\right)^{2}\left(\mathrm{t}_{\mathrm{i}}-\mathrm{t}_{\mathrm{i}-1}\right)+\left(\mathrm{t}_{\mathrm{i}}-\mathrm{t}_{\mathrm{i}-1}\right)^{2}
\end{gathered}
$$

Applying Lemma yields:

$$
\begin{aligned}
& \left.=\sum_{i=1}^{n} 3\left(t_{i}-t_{i-1}\right)^{2}-2\left(t_{i}-t_{i-1}\right)^{2}+\left(t_{i}-t_{i-1}\right)^{2}\right) \\
& =\sum_{i=1}^{n} 2\left(t_{i}-t_{i-1}\right)^{2} \leq 2 \Delta\left(\Pi^{(i)}\right) \sum_{i=1}^{n}\left(t_{i}-t_{i-1}\right)=2 \Delta\left(\Pi^{(i)}\right) 1
\end{aligned}
$$

For typographical case, set $\Delta^{(i)}=\Delta\left(\prod \varphi\right.$. If for instance:

$$
\Delta^{(i)}=\frac{\epsilon_{i}}{i^{2}}
$$

where, $\epsilon \rightarrow 0$ and then:

$$
\mathrm{P}\left[\left|\mathrm{Q}\left(\Pi^{(\mathrm{i})}\right)-1\right|>\mathrm{i} \sqrt{2 \Delta\left(\Pi^{(\mathrm{i})}\right)}\right]=\mathrm{P}\left[\left|\mathrm{Q}\left(\Pi^{(\mathrm{i})}\right)-1\right|^{2}>2 \epsilon_{\mathrm{i}}\right]
$$

Which by Chebychev's inequality is bounded by:

$$
\begin{aligned}
& \leq \frac{\mathrm{E}\left(\mathrm{Q}\left(\Pi^{(\mathrm{i})}\right)-1\right)^{2}}{2 \epsilon_{\mathrm{i}}} \\
& \leq 2 \frac{\Delta\left(\Pi^{(\mathrm{i})}\right)}{2 \epsilon_{\mathrm{i}}}=\frac{2 \epsilon_{\mathrm{i}}}{2 \epsilon_{\mathrm{i}} \mathrm{i}^{2}}=\mathrm{i}^{-2}
\end{aligned}
$$

Therefore;

$$
\sum_{i} \mathrm{P}\left[\left|\mathrm{Q}\left(\Pi^{(i)}\right)-1\right|>\mathrm{i} \sqrt{2 \Delta\left(\Pi^{(i)}\right)}\right]<\infty
$$

From the Borel-Cantelli lemma, we conclude:

$$
\mathrm{P}\left[\left|\mathrm{Q}\left(\Pi^{(i)}\right)-1\right|>i \sqrt{2 \Delta\left(\Pi^{(i)}\right)}\right] \text { i.o. }=0
$$

and for all large $\mathrm{i}$ (depending on the sample point $\mathrm{w}$ ),

$$
\left.\left|\mathrm{Q}\left(\Pi^{(\mathrm{i})}\right)-1\right| \leq \sqrt{2 \epsilon_{\mathrm{i}}}\right] \rightarrow 0
$$

as required.

Local maxima and points of increase: In this section, we prove some results on the irregular behaviour of the Brownian paths. We start with the following results.

Proposition: a.s., $t \rightarrow B_{t}(w)$ is monotone in no interval.

Definition: Let $\mathrm{f}:[0, \infty) \rightarrow \mathrm{R}$. A number $\mathrm{t} \geq 0$ is called a point of local maximum, if there exists a number $\delta>0$ with $f(s) \leq f(t)$, for every $s \in\left[(t-\delta)^{+}, t+\delta\right]$. t is called a point of strict local maximum if there exists $\delta>0$ with $\mathrm{f}(\mathrm{s})<\mathrm{f}(\mathrm{t})$ for every $\mathrm{s} \in\left[(\mathrm{t}-\delta)^{+}, \mathrm{t}+\delta\right] \backslash\{\mathrm{t}\}$.

Theorem: a.s., the set of points of local maximum of the Brownian path $t \rightarrow B_{t}(w)$ is countable and dense in $[0, \infty)$ and all local maxima are strict. The proof of theorem depends on the following facts:

- Let $\mathrm{f}:[0, \infty) \rightarrow \mathrm{R}$ be continuous. Then the set of points of strict local maximum for $f$ is countable. Further if $f$ is monotone in on interval then the set of points of local maximum is dense in $[0, \infty)$

- Let $\mathrm{X}, \mathrm{Y}$ and $\mathrm{Z}$ be independent random variables and $f: R^{3} \rightarrow[0, \infty)$ be measurable. Then:

$$
\mathrm{E} \mathrm{f}(\mathrm{X}, \mathrm{Y}, \mathrm{Z})=\int_{\mathrm{R}^{2}} \int \mathrm{Ef}(\mathrm{X}, \mathrm{y}, \mathrm{z}) \mu_{\mathrm{Y}}(\mathrm{dy}) \mu_{\mathrm{Z}}(\mathrm{dz})
$$

Where, $\mu_{\mathrm{Y}}(\mathrm{dy})$ and $\mu_{\mathrm{Z}}(\mathrm{dz})$ distributions of $\mathrm{Y}$ and $\mathrm{Z}$, respectively. The proof of first is elementary. The results in second is well known. T o prove it, it is verified first for indicator functions of measurable rectangles in $\mathrm{R}^{3}$ then for indicators of measurable sets then for simple functions and finally for non-negative measurable functions.

Proof of theorem: Because of fact above and proposition it suffices to show that for almost every Brownian path, all local maxima are strict:

$$
\text { Let } A_{t_{1} t_{2} t_{3} t_{4}}=\left\{w: \max _{t_{3} \leq t \leq t_{4}} B_{t}(w)-\max _{t_{1} \leq t \leq t_{2}} B_{t}(w) \neq 0\right\}
$$

Clearly, $A_{t 1+2+3}$ is measurable as is the set A defined by:

$$
A=\cap A_{t 12+35 t 4}
$$

Where, the intersection is taken over all quadruples of rational numbers $\left(t_{1}, t_{2}, t_{3}, t_{4}\right)$ satisfying $0 \leq t_{1}<t_{2}<t_{3}<t_{4}$. 
It is easily verified that if $\mathrm{w} \in \mathrm{A}$ then all local maxima of $t \rightarrow B_{t}(w)$ are strict. Hence to prove the theorem, it suffices to show that:

$$
\begin{aligned}
& P\left(A_{t_{1} t_{2} t_{3}}\right)=1 \text { but } \max _{t_{3} \leq t \leq t_{4}} B_{t}(w)-\max _{t_{1} \leq t \leq t_{2}} B_{t}(w)=\left(B_{t_{3}}(w)-B_{t_{2}}(w)\right)+ \\
& \min _{t_{1} \leq t \leq t_{2}}\left[B_{t_{2}}(w)-B_{t}(w)\right]+\max _{t_{3} \leq t \leq t_{4}}\left[B_{t}(w)-B_{t_{3}}(w)\right]
\end{aligned}
$$

Where, the three random variables appearing in the RHS of the above equality are independent. By fact above:

$$
P\left(A_{t_{1} t_{2} t_{3}}\right)=\iint_{R^{2}} P\left[B_{t_{3}}-B_{t_{2}}+y+z \neq 0\right] \mu_{1}(d y) \mu_{2}(d z)
$$

where, $\mu_{1}(d y)$ and $\mu_{2}(d z)$ are the distributions of:

$$
\min _{t_{1} \leq t \leq t_{2}}\left[B_{t_{2}}-B_{t}\right] \text { and } \max _{t_{3} \leq t \leq t_{4}}\left[B_{t}-B_{t_{3}}\right]
$$

respectively. But $P\left[B_{13}-B_{12}+y+Z \neq 0\right] \equiv 1$. It follows that $\mathrm{P}\left(\mathrm{A} \mathrm{t}_{1}, \mathrm{t}_{2}, \mathrm{t}_{3}\right)=1$.

The Law of the Iterated Logarithm (LIL): Khintchine's law of the iterated logarithm for BM is a very precise statement about how BM oscillates in a neighbourhood of the origin:

$$
\text { Let h(t) }=\sqrt{2 t \log \log t^{-1}}
$$

We have the following theorem.

Theorem: For standard BM B(t):

$$
P\left[\limsup \frac{B(t)}{h(t)}=1\right]=1 \text { and } P\left[\liminf _{t \downarrow_{0}} \frac{B(t)}{h(t)}=-1\right]=1
$$

Remark: The second statements follows from the first. Since $B(.)^{d}=-B($.$) , the first result yields:$

$$
\begin{aligned}
& \underset{t \downarrow_{0}}{\limsup } \frac{-B(t)}{h(t)}=1 \text { almost surely that is } \\
& -\limsup _{t \downarrow_{0}}-\frac{B(t)}{h(t)}=\limsup _{t \downarrow_{0}} \frac{B(t)}{h(t)}=-1 \text { almost surely }
\end{aligned}
$$

Remark: Khintchine's law also gives us a LIL near infinity:

$$
\begin{aligned}
& P\left[\limsup _{t \rightarrow \infty} \frac{B(t)}{\sqrt{2 t \log \log t}}=1,\right. \\
& \left.\underset{t \rightarrow \infty}{\liminf } \frac{B(t)}{\sqrt{2 t \log \log t}}=-1\right]=1
\end{aligned}
$$

This follows from the time reversal property of BM. We have:

$$
\mathrm{B}^{(1)}(t)= \begin{cases}\mathrm{tB}(1 / \mathrm{t}), & \text { if } \mathrm{t}>0 \\ 0, & \text { if } \mathrm{t}=0\end{cases}
$$

is a BM so, almost surely:

$$
\begin{aligned}
1 & =\underset{t \downarrow_{0}}{\limsup } \frac{B^{(1)}(t)}{h(t)} \\
& =\limsup _{t \downarrow_{0}} \frac{t B(1 / t)}{\sqrt{2 t \log \log t^{-1}}}
\end{aligned}
$$

Let $\mathrm{t}=1 / \mathrm{s}$ so, $\mathrm{s} \rightarrow \infty$ we find:

$$
\begin{aligned}
& \limsup _{s \rightarrow \infty} \frac{\frac{B(s)}{s}}{\sqrt{2 \frac{1}{s} \log \log s}} \\
& =\limsup _{s \rightarrow \infty} \frac{B(s)}{\sqrt{2 s \log \log s}}=1 \text { almost surely }
\end{aligned}
$$

Remark: An important conclusion from the LIL is that almost all paths $\mathrm{B}(\mathrm{t}, \mathrm{w})$ of $\mathrm{BM}$ pass through 0 infinitely often in every neighbourhood of zero. Thus, for any $\epsilon>0$, $\delta>0, B(t, w)$ is infinitely often in the region $\{(t, y): 0 \leq t \leq \delta$, $(1-\epsilon) \mathrm{h}(\mathrm{t})<\mathrm{y}<(1+\epsilon) \mathrm{h}(\mathrm{t})\}$ and also infinitely often in the region $\{(\mathrm{t}, \mathrm{y}): 0 \leq \mathrm{t} \leq \delta,-(1+\epsilon) \mathrm{h}(\mathrm{t})<\mathrm{y}<-(1-\epsilon) \mathrm{h}(\mathrm{t})\}$. Therefore, the path oscillates near 0 jumping from being positive to negative infinitely many times (Fig. 1). We need the following Lemma for the proof of the theorem.

Lemma: Suppose B is the standard BM. For $\alpha>0, \beta>0$ and any $t>0$, we have:

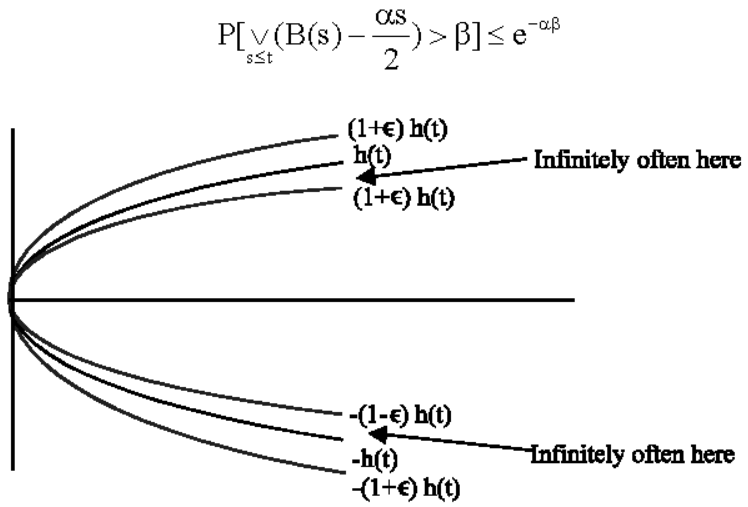

Fig. 1: Path osallation 


\section{Proof of the theorem}

Part 1: We show:

$$
\underset{t \downarrow 0}{\limsup } \frac{B(t)}{h(t)} \leq 1
$$

For $0<\theta<1,0<\boldsymbol{\delta}<1$, let $\mathrm{t}_{\mathrm{n}}=\boldsymbol{\theta}^{\mathrm{n}-1}$

$$
\alpha_{\mathrm{n}}=\frac{(1+\delta)}{\theta^{\mathrm{n}}} \mathrm{h}\left(\theta_{\mathrm{n}}\right), \beta_{\mathrm{n}}=\frac{1}{2} \mathrm{~h}\left(\theta^{\mathrm{n}}\right)
$$

Thus:

$$
\begin{gathered}
\alpha_{\mathrm{n}} \beta_{\mathrm{n}}=\frac{(1+\delta)}{\theta^{\mathrm{n}}} \frac{\mathrm{h}^{2}\left(\theta^{\mathrm{n}}\right)}{2} \\
=\frac{(1+\delta) 2 \theta^{\mathrm{n}} \log \log \theta^{-\mathrm{n}}}{2 \theta^{\mathrm{n}}} \\
=(1+\delta) 2 \theta^{\mathrm{n}} \log \log \theta^{-\mathrm{n}} \\
=\log \left(\log \theta^{-\mathrm{n}}\right)^{1+\delta} \\
\mathrm{e}^{-\alpha_{\mathrm{n}} \beta_{\mathrm{n}}}=\mathrm{e}^{-\log \left(\log \theta^{-\mathrm{n}}\right)^{1+\delta}} \\
\frac{1}{\left(\log \theta^{-\mathrm{n}}\right)^{1+\delta}}=\left(\frac{1}{\mathrm{n} \log \theta^{-1}}\right)^{1+\delta} \\
=\frac{\mathrm{K}}{\mathrm{n}^{1+\delta}}
\end{gathered}
$$

for a constant $\mathrm{K}>0$ then applying Lemma, we have:

$$
\begin{aligned}
& P\left[\underset{s \leq t_{n}}{\vee}\left[B(s)-\frac{\alpha_{n} s}{2}\right]>\beta_{n}\right] \leq e^{-\alpha_{n} \beta_{n}}=\frac{K}{n^{1+\delta}} \\
& \sum_{n} P\left[\underset{s \leq t_{n}}{\vee}\left[B(s)-\frac{\alpha_{n} s}{2}\right]>\beta_{n}\right]<\infty
\end{aligned}
$$

By the Borel-Cantelli lemma ultimately for all sufficiently large (depending on $\mathrm{w}$ ) $\mathrm{n}$ :

$$
\underset{s \leq t_{n}}{\vee}\left[B(s)-\frac{\alpha_{n} s}{2}\right] \leq \beta_{n}
$$

For such n we have:

$$
\underset{s \leq t_{n}}{\vee} B(s)-\frac{\alpha_{n} t_{n}}{2} \leq \beta_{n}
$$

Paraphrasing the previous statement, we have:

$$
\underset{s \leq t_{n}}{\vee} B(s) \leq \beta_{n}+\frac{\alpha_{n} t_{n}}{2}
$$

For such $n, t \in\left[\theta^{n}, \theta^{n-1}\right)=\left[t_{n+1}, t_{n}\right)$, we have:

$$
\begin{aligned}
& \mathrm{B}(\mathrm{t}) \leq \underset{s \leq t_{\mathrm{n}}}{\vee} \mathrm{B}(\mathrm{s}) \leq \beta_{\mathrm{n}}+\frac{\alpha_{\mathrm{n}} \mathrm{t}_{\mathrm{n}}}{2} \\
& \frac{\mathrm{h}\left(\theta_{\mathrm{n}}\right)}{2}+\frac{(1+\delta)}{2 \theta^{\mathrm{n}}} \mathrm{h}\left(\theta_{\mathrm{n}}\right) \theta^{\mathrm{n}-1} \\
& \mathrm{~h}\left(\theta_{\mathrm{n}}\right)\left[\frac{1}{2}+\frac{(1+\delta)}{2 \theta}\right]
\end{aligned}
$$

Using the fact that $h(t)$ is non-decreasing in a neighbour hood of 0 , we get the bound:

$$
\leq \mathrm{h}(\mathrm{t})\left[\frac{1}{2}+\frac{(1+\delta)}{2 \theta}\right]
$$

We may conclude that for any $0<\theta<1$ and $0<\delta<1$ :

$$
\underset{\mathrm{t} \downarrow 0}{\limsup } \frac{\mathrm{B}(\mathrm{t})}{\mathrm{h}(\mathrm{t})} \leq\left[\frac{1}{2}+\frac{(1+\delta)}{2 \theta}\right]
$$

Observe that the left side is independent of $\theta$ and $\delta$, so in right side, we let $\delta \downarrow 0, \theta \dagger 1$. This gives desired assertion for part 1 .

Part 2: We show:

$$
\limsup _{t \downarrow_{0}} \frac{B(t)}{h(t)} \geq 1
$$

Define independent events:

$$
A_{n}=\left[B\left(\theta^{n}\right)-B\left(\theta^{n+1}\right) \geq(1-\sqrt{\theta}) h\left(\theta^{n}\right)\right]
$$

Thus:

$$
\mathrm{PA}_{\mathrm{n}}=\mathrm{P}\left[\begin{array}{l}
\frac{\mathrm{B}\left(\theta^{\mathrm{n}}\right)-\mathrm{B}\left(\theta^{\mathrm{n}+1}\right)}{\sqrt{\theta^{\mathrm{n}}(1-\theta)}} \\
>(1-\sqrt{\theta}) \sqrt{\frac{2 \theta^{\mathrm{n}} \log \log \theta^{-\mathrm{n}}}{\theta^{\mathrm{n}}(1-\theta)}}
\end{array}\right]
$$

The random variable:

$$
\frac{\mathrm{B}\left(\theta^{\mathrm{n}}\right)-\mathrm{B}\left(\theta^{\mathrm{n}+1}\right)}{\sqrt{\theta^{\mathrm{n}}(1-\theta)}}
$$

is $\mathrm{N}(0,1)$. Call the right side of the inequality above $\mathrm{x}_{\mathrm{n}}$. Since $\theta^{-n} \rightarrow \infty$, we have $x_{n} \rightarrow \infty$. Applying Mill's ratio, we have:

$$
\mathrm{PA}_{\mathrm{n}}=\mathrm{P}\left[\mathrm{N}(0,1)>\mathrm{x}_{\mathrm{n}}\right] \sim \frac{\mathrm{n}\left(\mathrm{x}_{\mathrm{n}}\right)}{\mathrm{x}_{\mathrm{n}}}
$$

$$
=\frac{\frac{1}{\sqrt{2 \pi}} \mathrm{e}^{-\frac{1}{2}\left[\frac{1-\sqrt{\theta}}{\sqrt{1-\theta}} \log \log \theta^{-n}\right]^{2}}}{\frac{1-\sqrt{\theta}}{\sqrt{1-\theta}} \sqrt{\log n}}
$$




$$
\frac{\operatorname{Kexp}\left\{-\frac{1}{2}\left[\frac{1-\sqrt{\theta}}{\sqrt{1-\theta}}\right]^{2}\left(2 \log \log \theta^{-\mathrm{n}}\right\}\right.}{\sqrt{\log n}}
$$

Since:

$$
\frac{1-\sqrt{\theta}}{\sqrt{1-\theta}}<1
$$

We obtain the lower bound:

$$
\begin{aligned}
& \geq \frac{\mathrm{K}}{\sqrt{\log n}} \mathrm{e}^{\log \log \theta^{-n}} \\
& =\frac{\mathrm{K}^{\prime}}{\mathrm{n} \sqrt{\log \mathrm{n}}}
\end{aligned}
$$

We conclude that $\sum_{\mathrm{n}} \mathrm{Pa}_{\mathrm{n}}=\infty$ and by the BorelCantelli lemma, we get $\mathrm{P}\left\{\mathrm{A}_{\mathrm{n}}\right.$ i.o $\}=1$. Thus, for infinitely many $n$, we have:

$$
\begin{aligned}
& \mathrm{B}\left(\theta^{\mathrm{n}}\right)-\mathrm{B}\left(\theta^{\mathrm{n}+1}\right)>(1-\sqrt{\theta}) h\left(\theta^{\mathrm{n}}\right) \\
& \text { or equivalently, } \mathrm{B}\left(\theta^{\mathrm{n}}\right)>\mathrm{B}\left(\theta^{\mathrm{n}+1}\right)+(1-\sqrt{\theta}) h\left(\theta^{\mathrm{n}}\right)
\end{aligned}
$$

From part 1 of this proof, we have that $\mathrm{B}(\mathrm{t}) \leq 2 \mathrm{~h}(\mathrm{t})$ for small t. Since $-\mathrm{B}(\mathrm{t})$ is also $\mathrm{BM}$, we have that $-\mathrm{B}(\mathrm{t}) \geq-2 \mathrm{~h}(\mathrm{t})$. For $\mathrm{n}$ sufficiently large, $\mathrm{B}\left(\theta^{\mathrm{n}+1}\right) \geq 2 \mathrm{~h}\left(\theta^{\mathrm{n}+1}\right)$. For infinitely many $n$ :

$$
\mathrm{B}\left(\theta^{\mathrm{n}}\right)>(1-\sqrt{\theta}) \mathrm{h}\left(\theta^{\mathrm{n}}\right)-2 \mathrm{~h}\left(\theta^{\mathrm{n}+1}\right)
$$

Thus for infinitely many sufficiently large n, we have:

$$
\begin{aligned}
& \frac{\mathrm{B}\left(\theta^{\mathrm{n}}\right)}{\mathrm{h}\left(\theta^{\mathrm{n}}\right)}>1-\sqrt{\theta}-\frac{2 \mathrm{~h}\left(\theta^{\mathrm{n}+1}\right)}{\mathrm{h}\left(\theta^{\mathrm{n}}\right)} \mathrm{h}\left(\theta^{\mathrm{n}}\right) \\
& =1-\sqrt{\theta}-2 \sqrt{\frac{2 \theta^{\mathrm{n}+1} \log \log \theta^{-(\mathrm{n}+1)}}{2 \theta^{\mathrm{n}} \log \log \theta^{-\mathrm{n}}}} \\
& =1-\sqrt{\theta}-2 \sqrt{\theta(1+\mathrm{O}(1))} \\
& >1-\sqrt{\theta}-3 \sqrt{\theta}=1-4 \sqrt{\theta}
\end{aligned}
$$

The conclusion is that:

$$
\limsup _{t \downarrow_{0}} \frac{B(t)}{h(t)} \geq 1-4 \sqrt{\theta}
$$

Note that the left side is independent of $\theta$ so that on the right side we may let $\theta \downarrow 0$ to obtain the required result.

Recent developments: Finally, we see a recent theoretical development. A Brownian path in two dimensions can be produced from two independent one dimensional Brownian functions $\mathrm{X}(\mathrm{t})$ and $\mathrm{Y}(\mathrm{t})$. Suppose, we consider the range $0 \leq t \leq T$ and $X(0)=Y(0)=0$. Then the path $(\mathrm{X}(\mathrm{t})-(\mathrm{t} / \mathrm{T}) \mathrm{X}(\mathrm{T}), \mathrm{Y}(\mathrm{t})-(\mathrm{t} / \mathrm{T}) \mathrm{Y}(\mathrm{T}))$ begins and ends at the origin and is called a Brownian cluster. Visual comparisons between Brownian cluster simulations and the coastlines of islands led Benoit Mandelbrot to conjecture that the periphery of a Brownian cluster (the part can be reached from far away without crossing any other point of the cluster) has dimension $4 / 3$. This has been recently proved.

\section{CONCLUSION}

In this study, we discussed a theoretical study of the enhanced path properties of a standard Brownian motion and the law of the iterated logarithm about how Brownian motion oscillates in a neighbourhood of the origin i.e., zero.

\section{REFERENCES}

Doob, J.L., 1953. Stochastic Processes. Wiley, New York, pp: 107-118.

Ito, K. and H.P. McKean, 1965. Diffusion Processes and their Sample Paths. Springer-Verlag, Berlin, New York, pp: 306-312.

Karatzas, I. and S.E. Shreve, 1988. Brownian Motion and Stochastic Calculus. Springer-Verlag, New York.

Levy, P., 1965. Processus Stochastiques et Mouvement Brownien. Gautheir Villars, Paris.

McKean, H.P., 1969. Stochastic Integrals. Academic Press, New York, London. 